\title{
Thanatophoric dysplasia: A review
}

\author{
H Wainwright, $\mathrm{MB} \mathrm{ChB}$, FFPath (SA) \\ Division of Anatomical Pathology, Department of Pathology, Faculty of Health Sciences, University of Cape Town and Groote Schuur Hospital, \\ Cape Town, South Africa
}

Corresponding author: H Wainwright (helen.wainwright@uct.ac.za)

\begin{abstract}
Thanatophoric dysplasia is a well-known cause of potentially lethal short-limbed dwarfism in the newborn. The diagnosis is usually made by the recognition of characteristic radiological changes and confirmed at autopsy by demonstration of specific morphological and histological changes in the brain. This review is based upon the author's personal experience and archived data of 19 cases and concerns the clinical and radiographic manifestations, autopsy findings, molecular pathogenesis and the approach to antenatal diagnosis.
\end{abstract}

S Afr Med J 2016;106(6 Suppl 1):S50-S53. DOI:10.7196/SAMJ.2016.v106i6.10993

Thanatophoric dysplasia (TD) is the most common neonatal lethal skeletal dysplasia, and it is regularly encountered in the context of antenatal diagnosis and autopsy. This review of the disorder is based upon personal experience and data concerning 19 cases archived in the Division of Pathological Anatomy, University of Cape Town (UCT).

Professor Peter Beighton had a special interest in genetic bone disorders and following his appointment at UCT in 1972 he established a special clinic for affected persons at the Princess Alice Orthopaedic Hospital. He also undertook diagnostic screening at facilities for physically handicapped persons throughout South Africa. These activities led to the publication of his early monographs ${ }^{[1-3]}$ and to his involvement in the International Classification of Osteochondrodysplasias. ${ }^{[4]}$ After mandatory retirement in 1999, he extended his interest in skeletal dysplasias to neonates, stillbirths and fetuses and assisted me with my research in this field. Together we have co-authored 11 articles concerning the most severe and lethal skeletal dysplasias. In early intrauterine life, the radiological features may be poorly developed and the pathological changes in the bones more difficult to determine than in a full-term pregnancy. Information from prenatal ultrasonic imaging from 12 weeks' gestation onwards is now available and it is invaluable in the early detection and diagnosis of a specific skeletal dysplasia in order to facilitate appropriate obstetrical and genetic management.

The incidence of type $1 \mathrm{TD}$ (TD1) is variously quoted as 1 in $20000-40000$ stillborn and liveborn infants (MIM 187600) ${ }^{[5]}$ or 1 in 33000 - 47000 live births $\left.{ }^{[6]}\right]$. The condition was initially termed thanatophoric dwarfism in $1967,{ }^{[7]}$ and a decade later the name was changed to thanatophoric dysplasia. The name was derived from the Greek word thanatos meaning 'death' and phoros meaning 'provoking'.

\section{Manifestations}

TD is traditionally divided into two forms on a basis of the radiographic appearances - TD1 with curved femora and usually a normal skull and type 2 (TD2) with straight femora and frequently a trilobal clover-leaf skull.

Phenotypically, there is marked symmetrical shortening of the limbs with redundant skin folds, shortening of hands and feet, macrocephaly with frontal bossing, a depressed nasal bridge, small chest, large protuberant abdomen, and a trunk of relatively normal length (Fig. 1). The radiological features of TD1 are characteristic when the infant is delivered at term - macrocephaly, symmetrical micromelic shortening of long bones, metaphyseal cupping of the

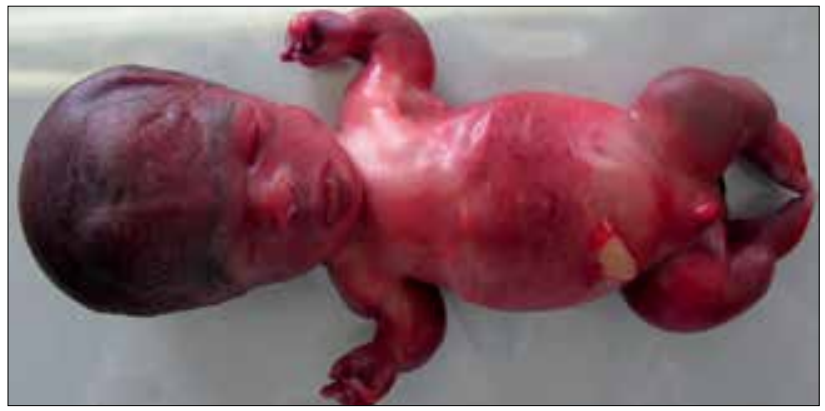

Fig. 1. Immature fetus with TD1 with macrocephaly, frontal bossing, depressed nasal bridge, small chest, protuberant abdomen and short symmetrical bowed limbs.

proximal femora, telephone-receiver shaped femora, hypoplastic or small scapulae, platyspondyly with $\mathrm{H}$-shaped vertebrae in the anterior-posterior view, a narrow thorax with short ribs, and characteristic triradiate acetabulum with short sacrosciatic notches. Interpediculate narrowing of the spinal canal evident on lateral views results in damage to the spinal cord in rare survivors. In TD2, the skull has a marked anterior depression forming the trilobal cloverleaf skull. ${ }^{[8]}$

Bone radiographic morphometry shows that longitudinal bone growth is not equally inhibited across the growth plate, resulting in the lateral spurs at the metaphyseal growth plates. The platyspondyly is due to a reduced height of the anterior ossification centres of the vertebral bodies, while laterally there is no restriction in growth. Interplay between mechanical factors and the effects of the underlying mutation are additional factors in this process. ${ }^{[9]}$

\section{Autopsy findings}

At postmortem, the head is macrocephalic due to macroencephaly and symmetrical deep grooves are present on the basal surfaces of the temporal lobes of the brain. Histologically, the ends of the long bones show markedly disordered growth plates. The normal growth plate has three zones - the resting zone with disordered chondrocytes and abundant matrix, the proliferative zone, and the hypertrophic zone where the chondrocytes die. Below the hypertrophic zone is the zone of provisional calcification, then the primary and secondary spongiosum. In TD1, the resting zone is uninvolved. The proliferative and hypertrophic zones show variable disorganisation with complete absence in some areas and 
focal columns in others. A typical horizontal band extends from the perichondrial fibrous tissue at the periphery into the growth plate and there is transformation of this mesenchymal tissue into irregular, poorly orientated bony trabeculae. The cartilaginous bars are reduced in number and are thin and distorted. Perichondrial ossification extends above the level of endochondral ossification. The value of histological assessment of the growth plate in the diagnosis of TD1 has been known since 1979 (Fig. 2). ${ }^{[10]}$

The ultrasonographic, radiological and histological features in fetuses and newborns are well documented. ${ }^{[11]}$ Young fetuses pose a special challenge to diagnosis as diagnostically characteristic radiological features may be absent. Ultrasound shows severely shortened and bowed long bones, a narrow thorax filled by the heart, a protuberant abdomen and bossing of the skull. In young fetuses, the ultrasonographic diagnosis may not be specific. Nevertheless, detection of severe shortening of limbs with bowing and severe thoracic hypoplasia suggest a lethal skeletal dysplasia with pulmonary

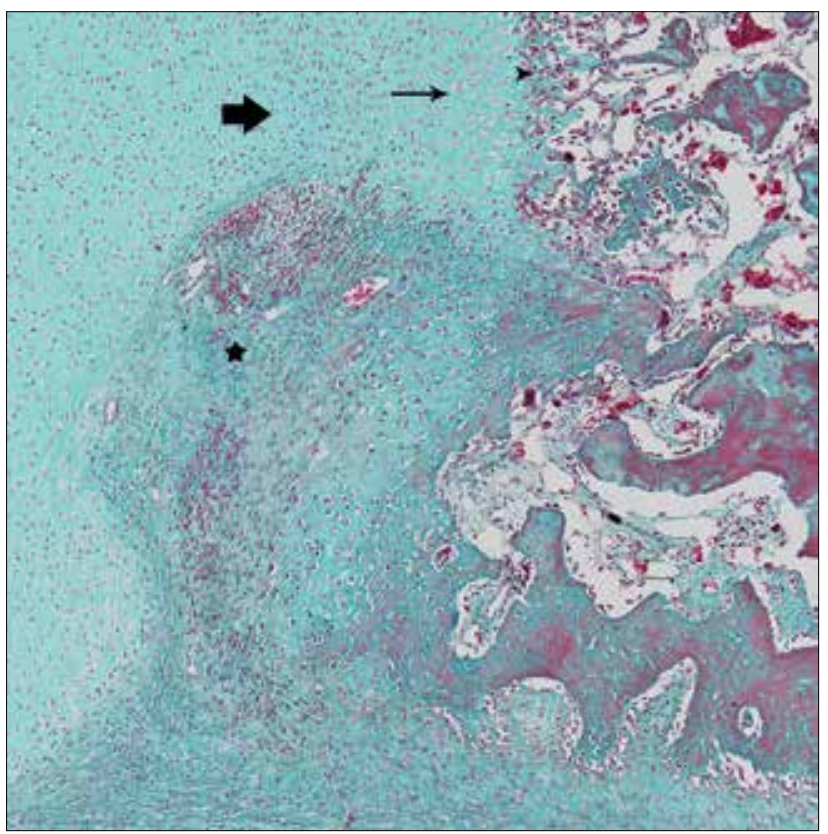

Fig. 2. Masson's trichrome stain shows histology of the markedly disordered growth plate with a horizontal band extending into the growth plate indicated by a star; the short arrow shows the proliferative zone, the long arrow shows the short chondrocyte columns - the arrowhead shows the disordered metaphysis.

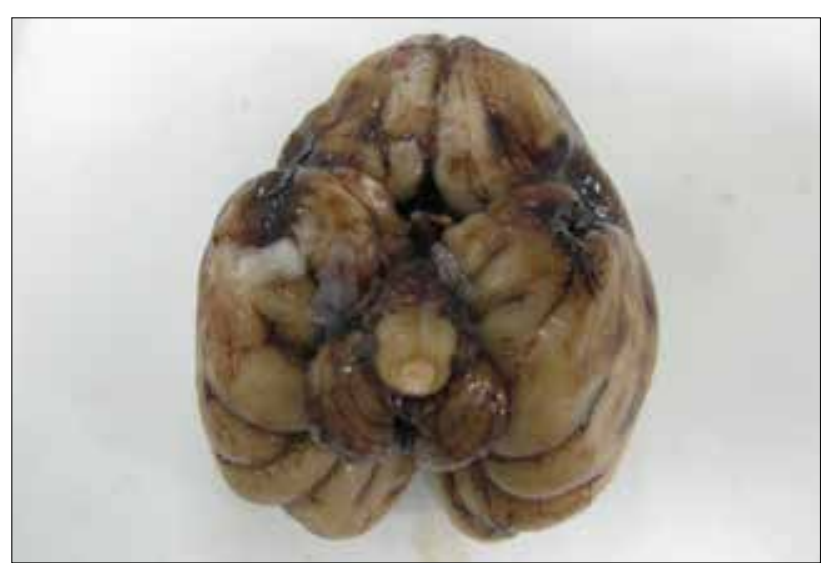

Fig. 3. Inferior surface of the brain with the basal surfaces of the temporal lobes showing deep symmetric grooves diagnostic of TD1. hypoplasia, which is usually a sufficient indication for termination of pregnancy.

The central nervous system abnormality is not secondary to the bone changes in the skull, such as synostosis which occur late in gestation, but due to the underlying FGFR3 mutation. Fibroblast growth factors (FGFs) 1 - 4 are expressed in the developing brain. Using a mouse model, it has been shown that FGFR3 is involved in area patterning, progenitor proliferation and a reduction in apoptosis within the brain. The most typical neuropathological changes are megaloencephaly, dysplasia of the hippocampus, rudimentary dentate nucleus, polymicrogyria, temporal lobe hyperplasia, 1 5 abnormal deep transverse sulci on the basal surface, subependymal neuronal heterotopia and subarachnoid neuroglial heterotopia (Fig. 3). ${ }^{[12]}$ Temporal lobe ventriculomegaly is common, but severe hydrocephalus occurs only in $18 \%$ of cases due to a small foramen magnum. The cerebellum may be hypoplastic or normal. The diagnostic basal transverse sulci are seen at 20 weeks' gestation when the brain normally has a smooth surface. The abnormal sulcation on the medial aspects of both temporal and occipital lobes has been identified on antenatal sonography at 21 weeks and confirmed on antenatal MRI, postnatal MRI and at postmortem. ${ }^{[13]}$

\section{Molecular pathogenesis}

The inception of the molecular age brought major changes to the former categorisation of skeletal dysplasias which has now been supplemented or replaced by a molecular classification. ${ }^{[14]}$ TD1 now falls under the broad group of the Fibroblast Growth Factor Receptor 3 (FGFR3) family [FGFR3, OMIM 134934]. The first activating or gain-in-function mutation in FGFR3 receptor tyrosine kinase that binds fibroblast growth factors was discovered in 1994 in achondroplasia. ${ }^{[15,16]}$ Other conditions associated with mutations in this gene include TD2. Numerous somatic mutations that cause diverse conditions such as specific skeletal dysplasias, skin disorders and malignancies have also been identified.

The FGFR3 gene encodes a protein, predominantly in boneproducing cells, which straddles the cell membrane with the inner end within the cell. The outer end projects from the surface and growth factors attach to it, resulting in its activation and action on bone. The gene regulates skeletal development by degradation of the bone morphogenetic protein (BMP) type 1 receptor. It has a negative effect on chondrogenesis/endochondral bone growth via inhibition of BMPR1a, which is required for chondrocyte differentiation. ${ }^{[17]}$

TD1 may result from a single amino acid substitution in either the extracellular or intracellular domains of the protein. Examples involving the extracellular domain include p.R248C (c.742C>T); p.Y373C $(\mathrm{c} .1118 \mathrm{~A}>\mathrm{G}){ }^{[18,19]}$ Stop codon mutations have been reported: p.X807G (c.2419T>G); p.x807R (c.2419T>A); p.X807C (c.2421A>T) and these mutations result in elongation of the protein. ${ }^{[20]}$

Both $K 650 M$ and K650E mutations in the FGFR3 gene show abnormal cellular location to the endoplasmic reticulum ${ }^{[21]}$ and they activate downstream signalling intermediates such as ERKMAP kinase. ${ }^{[22]}$ These two mutations can be recognised by light microscopy as large cytoplasmic inclusion bodies that stain positively with antibodies to FGFR3 protein $^{[23]}$ Other mutants such as $R 248 C$ and Y373C signal from the cell membrane and cause severe skeletal dysplasia. ${ }^{[24]}$

Numerous studies have involved investigations of mutations in TD1. The largest series consisted of 91 cases of TD1 and TD2 with FGFR3 mutations identified in the International Skeletal Dysplasia Registry (ISDR). ${ }^{[25]}$ The most common mutation was $R 248 C$, occurring in $50 \%$ of cases of TD1, followed by $Y 373 C$ in 
20\%. These cases had more severe radiological manifestations than TD1 with $R 248 C$, but there was phenotypic overlap. Nineteen cases with TD2 from the ISDR had the K650E mutation and showed better preservation of the growth plate compared with TD1. Two mutations in the FGFR3 gene result in virtually all cases of achondroplasia, which is allelic with TD2. The N540K mutation alone results in the less severe phenotype of hypochondrodyplasia.

In 2014, Xue, et al. ${ }^{[26]}$ reported an update from the ISDR in which mutation analysis involved sequencing of the entire coding region in 324 cases, including achondroplasia and hypochondroplasia. This extensive series showed that $90 \%$ of TD1 mutations were either pArg248Cys (66\%) or pTyr373Cys. The third most common was a stop codon mutation pX807 and the fourth was pSer249Cys. The mutation $p$ Glu370Cys accounted for $2.3 \%$ and pLys650Met for $1.2 \%$. This information is extremely useful when designing and costing commercial tests for TD1.

Mutations cause activation of FGFR3 by different mechanisms. Extracellular FGF ligands form dimers. The TD1 mutation Y373C forms covalent bound dimers between cysteine residues near the juxtamembrane domain. Amino acid substitutions in the intracellular domain, such as K650M in TD 1/SADDAM or K650E in TD2, mimic conformational changes resulting in dimerisation and

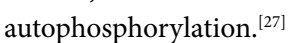

Low levels of activity require FGF ligand for activation: high levels of activity, as occurs with $R 248 \mathrm{C}$ and Y373C mutations, result in spontaneous dimerisation and are independent of ligand. Different amino acid substitutions cause differing degrees of activation of FGFR3, which translates into varying levels of chondrocyte inhibition. The most severe inhibition will result in the most severe degree of skeletal dysplasia.

In the absence of a mutation, the inhibition of chondrocytes via FGFR3 can be regarded as senescence, which can be experimentally reversed. Elsewhere in the body this senescence represents a natural barrier against tumour development. In embryogenesis, newly formed chondrocytes trigger strong initiation of FGFR3 expression as required for differentiation.

FGFR3 is a physiological negative regulator of bone growth. In humans, loss of function of FGFR 3 causes an autosomal recessive syndrome CATSHL, which is characterised by camptodactyly, hearing loss and skeletal overgrowth. ${ }^{[28]}$ Somatic mutations identical to those associated with TD1 can cause skin overgrowth or cancer, demonstrating that the TD mutation can act as an oncogene. ${ }^{[29]}$ The growth inhibitory role of FGFR3 in cartilage is unique in comparison to its aberrant signalling in other tissues. ${ }^{[30]}$

Aberrant signalling in the RAS/ERK pathway due to FRGF3 results in skeletal dysplasias. The signaling interferes with cyclin D3 complexes in the G1 phase of the cell cycle. There is an increase in cell cycle inhibitors that inhibit kinase activities. FGFR3 activation causes increased cell cycle inhibitors that prolong ERK activation only in chondrocytes so that the pathology is limited to the growth plate, as seen in skeletal dysplasias.

Animal studies in mice have produced an equivalent model to TD1 by introducing the $S 365 \mathrm{C}$ mutation into exon 10 of mouse FGFR3. However, the mutant mouse has severe dwarfism but no pulmonary hypoplasia, and the condition is therefore non-lethal. Other mouse models include short stature and a dome-shaped skull when the K6434E mutation is introduced into FGFR3. This mouse has a small thoracic cage and lethality. Mutation G369C provides an animal model for achondrogenesis. Regular subcutaneous injections of parathyroid hormone (PTH) permit a larger thorax to develop, thereby preventing pulmonary hypoplasia and causing an increase in length of long bones and a round head in the mouse models. ${ }^{[31]}$
For these reasons, mouse models can be used for testing potential therapies.

\section{Antenatal diagnosis in TD}

Early first-trimester prenatal diagnosis of skeletal dysplasia requires expertise. At 12 weeks, there may be subtle features such as frontal bossing, rarely an increase in nuchal translucency and short limbs. Early in the second trimester, a small thorax and short, bowed long bones may be demonstrated particularly by $2 \mathrm{D}$ and $3 \mathrm{D}$ ultrasound imaging. Ultrasound alone provides a specific antenatal diagnosis in $1 / 3-2 / 3$ of cases. ${ }^{[32]}$ The size of the thorax alone does not always indicate lethality, but parameters such as thoracic circumference and the thoracic-to-abdominal ratios may be more helpful. ${ }^{[33]}$

If the necessary facilities are available, mutational analysis during pregnancy is useful when TD is suspected. ${ }^{[34]}$ The FGFR3 gene consists of 19 exons and 18 introns spanning $16.5 \mathrm{~kb}^{[35]}$ and screening for the most frequently occurring mutations is cost effective.

Genotyping of mutations can be performed by high-resolution melting analysis. R248C is the most common mutation. Exon 7 was analysed in 10 samples with TD1 and 30 controls using melting curve analysis with a high-resolution melting instrument. This mutation was present in all 10 samples, giving a sensitivity and specificity of $100 \% \cdot{ }^{[36]}$

Other strategies for molecular testing use hot-spot exons 7,10 , 15 and 19 analysis with $80-90 \%$ sensibility. If hot spot exons are negative, then a search for rare mutations using sequential analysis of FGFR3 gene is performed. ${ }^{[37]}$ The nucleotide sequence of FGFR3 is highly conserved between man and mouse.

\section{Future prospects}

In the context of the FGFR3 group of disorders, postnatal treatment of achondroplasia is becoming a possibility in 2015 in First-World countries, raising hope for therapeutic intervention in certain skeletal dysplasias. Nevertheless, therapy for TD remains highly unlikely at the present time.

\section{References}

1. Beighton P. Inherited Disorders of the Skeleton. Edinburgh: Churchill Livingstone; 1978

2. Beighton P, Cremin B. Sclerosing Bone Dysplasias. Heidelberg: Springer-Verlag; 1980.

3. Horan F, Beighton P. Orthopaedic Problems in Inherited Skeletal Disorders. Heidelberg: SpringerVerlag; 1982

Beighton P, Giedion A, Gorlin R, et al. International classification of osteochondrodysplasia. International Working Group on Constitutional Diseases of Bone. Am J Med Gen 1992;44(2):223-229. 5. Oriola IM, Castilla EE, Barbosa-Neto JG. The birth prevalence rates for sketal dysplasia. J Med Genet 1982;23(4):328-332.

6. Waller DK, Correa A, Vo TM, et al. The population-based prevalence of achondroplasia and thanatophoric dysplasia in selected regions of the US. Am J Med Genet A 2008;146A:2385-2389. DOI:10.1002 dysplasia in sel

DOI:10.1002/ajmg.a.32485
Maroteaux,P, Lamy M, Robert JM. Le nanisme thanatophore. Presse Med 1967;75:2519-2514

7. Maroteaux,P, Lamy M, Robert JM. Le nanisme thanatophore. Presse Med 1967;75:2519-2514.
8. Kozlowski K, Beighton P. Gamut Index of Skeletal Dysplasias. An Aid to Radiodiagnosis. 3rd ed. 8. Kozlowski K, Beighton P. Gamut Index of Sk
Heidelberg: Springer-Verlag; 2001. p. 223-224.

9. Pazzaglia UE, Donzelli CM, Izzi C, et al. Thanatophoric dysplasia. Correlation among bone Xray morphometry, histopathology, and gene analysis. Skeletal Radiol 2014;43(9):1205-1215. DOI:10.1007/ s00256-014-1899-1

10. Sillence DO, Horton WA, Rimoin DL. Morphologic studies in the skeletal dysplasias. Am J Pathol 1979;96(3):813-870.

11. Brons JTJ, Van der Harten HJ. Skeletal Dysplasias. Pre- and Postnatal Identification. An Ultrasonographic, Radiologic and Pathologic Study. Amsterdam: Free University Hospital; p. 111-142. 12. Hevner RF. The cerebral cortex malformation in Thanatophoric Dysplasia - neuropathology and pathogenesis. Acta Neuropathol 2005;110:208-221.

13. Fink AM, Hingston T, Sampson A, et al. Malformation of fetal brain at 21 weeks fetal ultrasonography confirmed by antenatal MRI, postmortem imaging and autopsy. Pediatr Radiol 2010;40(Suppl 1):S134137. DOI: $10.1007 / 200247-010-1697-4$

14. Nikkels PGI. Diagnostic approach to congenital osteodysplasia at autopsy. Diagnostic Histopathology 2009;15-9 Mini-Symposium: Osteoarticular Pathology Elsevier Ltd

15. Shiang R, Thampson LM, Zhi YZ, et al. Mutation in the transmembrane domain of FRGF3 causes the most common form of dwarfism, achondroplasia. Cell 1994;78(2):335-342.

16. Rousseau F, Bonaventure J, Legai-Mallet L, et al. Mutation in the gene encoding fibroblast growth factor receptor 3 in achondroplasia. Nature 1994;371(6494):252-254.

17. Qi H, Jin M, Duan Y, et al. FGFR3 induces degradation of BMP type 1 receptor to regulate skeletal development. Biochim Biophys Acta 2014;1843(7):1237-1247.

18. Tavormina PL, Shiang R, Thompson LM, et al. Thanatophoric dysplasia (type 1 and 2) caused by distinct mutations in fibroblast growth factor receptor 3. Nat Genet 1995;9(3):321-328.

19. Kitoh H, Brodie SG, Kupke KG, et al. Lys650Met substitution in the tyrosine kinas domain of the fibroblast growth factor receptor 3 gene causes thanatophoric dysplasia Type 1. Mutations in brief no.199. Online. Hum Mutat 1998;12(5):362-363.

20. Rousseau F, el Ghouzzi V, Delezoide AL, et al. Missense FGFR3 Mutations create cysteine residues in thanatophoric dysplasia type. Hum Mol Genet 1996;5(4):509-512. 
21. Raffioni S, Zhu YZ, Bradshaw RA, et al. Effect of transmembrane kinase domain mutation on fibroblast growth factor receptor 3 chimera signaling in PC12 cells. A model for the control of receptor tyrosine growth factor receptor 3 chimera signaling in PC12 cells. A
kinase activity. J Biol Chem 1998;273(52):35250-35259.

22. Lievens PM, Liboi E. The thanatophoric dysplasia type II mutation hampers complete maturation of fibroblast growth factor receptor 3 (FGFR3) which activates transcription 1(STAT1) from the of fibroblast growth factor receptor 3 (FGFR3) which activa

23. Brodie SG, Kitoh H, Lachman RS, Nolasco LM, Mekikian PB, Wilcox WR. Platyspondylic lethal skeletal dysplasia, San Diego type, is caused by FGFR3 mutation. Am J Med Genet 1999;84(5):476-480. 24. Krejci P. The paradox of FGFR3 signaling in skeletal dysplasia: Why chondrocytes growth arrest while other cells overproliferate. Mutant Res Rev Mutat Res 2014;759:40-48. DOI:10.1016/j.mrrev.2013.11.001 25. Wilcox WR, Tavormina PL, Krakow D, et al. Molecular, radiologic and histologic correlation in thanatophoric dysplasia. Am J Med Genet 1998;78(3):274-281.

26. Xue Y, Sun A, Mekikia PB, et al. FGFR3 mutation frequency in 324 cases from the International Skeletal Dysplasia Registry. Mol Genet Genomic Med 2014;2(6):497-503. DOI:10.1002/mgg3.96

27. Webster MK, D'Avos PY, Robertson SC, et al. Profound ligand-independent kinase activation of FGFR3 by the activation loop mutation responsible for a lethal skeletal dysplasia, Thanatophoric Dysplasia Type II. Molec Cell Biol 1996;16(8):4081-4087.

28. Toydemir RM, Brassington AE, Bayrak-Toydemir P, et al. A novel mutation in FGFR3 causes camptodactyly tall stature, and hearing loss (CATSHL) syndrome. Am J Hum Genet 2006;79(5):935-941.DOI:10.1086/508433

29. Foldynova-Trantirkova S, Wilcox WR, Kreici P. Sixteen years \& counting: The current understanding of Fibroblast Growth Factor Receptor 3 signaling in skeletal dysplasia. Hum Mutat 2012;33(1):29-41. DOI:10.1002/humu. 21636
30. Krejci P. The paradox of FGFR3 signaling in skeletal dysplasia; why chondrocytes growth arrest while other cells proliferate. Mutat Res Rev Mutat Res 2014;759:40-48. DOI:10.1016/j mrrev.2013.11.001

31. Xie Y, Su N, Jin M, et al. Intermittent PTH (1-34) injection rescues the retarded skeletal dysplasia Xie $\mathrm{Y}, \mathrm{Su} \mathrm{N}$, Jin $\mathrm{M}$, et al. Intermittent $\mathrm{PTH}(1-34)$ injection rescues the retarded skeletal dysplasia
postnatal lethality of mice mimicking human achondroplasia and thanatophoric dysplasia. Hum Mol postnatal lethality of mice mimicking human achondrop
Genet 2012;21(18):3941-3955. DOI:10.1093/hmg/dds181

32. Parilla BV, Leeth EA, Kambich MP, Chilis P, MacGregor SN. Antenatal detection of skeletal dysplasia. J Ultrasound Med 2003;22(3):255-258

33. Wong HS, Kidd A, Zuccullo J, et al. A case of thanatophoric dysplasia. Early prenatal 2D and 3D sonographic findings and molecular confirmation of diagnosis. Fetal Diagn Ther 2008;24(1):71-73. DOI:10.1159/000132411

34. Chen CP, Chern SR, Wang W, Wang TY. Second-trimester molecular diagnosis of a heterozygous (R248D) mutation in the FGFR3 gene in a thanatophoric dysplasia variant following suspicious ultrasound findings. Ultrasound Obstet Gynecol 2001;17(3):272-273.

35. Perez-Castro AV, Wilson J, Altherr MR, et al. Genomic organization of the human fibroblast growth factor receptor 3 (FGFR3) geneand comparative sequence analysis with the mouse FGFR3 gene. Genomics 1997;41(1):10-16.

36. Liu YN, Li R, Li DZ Genotyping of the C742T mutation of the FGFR3 gene causing type 1 thanatophoric dysplasia by high-resolution melting analysis. J Matern Fetal Neonatal Med 2011;24(1):186-188. dysplasia by high-resolution melting
DOI:10.3109/14767058.2010.482621

37. Pazzaglia UE, Donzelli GM, Izzi C, et al. Thanatophoric dysplasia. Correlation among bone X-ray, morphometry, histopathology, and gene analysis. Skeletal Radiol 2014;43(9):1205-1215. 